\title{
A Non-contact Detection Method for Smelting in Submerged Arc Furnace based on Magnetic Field Radiation
}

\author{
WeiLing Liu and XiaoMing Chang* \\ College of Computer Science and Technology, Taiyuan University of Technology, No. 79 Yingze West Street, \\ Taiyuan 030024, Shanxi, PR China
}

(Received 21 February 2016, Received in final form 18 April 2016, Accepted 21 April 2016)

\begin{abstract}
This paper demonstrates the key parameter detection for smelting of submerged arc furnace (SAF) based on magnetic field radiation. A magnetic field radiation model for the inner structure of SAF is established based on relative theory of electromagnetic field. A simple equipment of $3 D$ magnetic field detection system is developed by theoretical derivation and simulation. The experiments are carried out under the environment of industrial field and AC magnetic field generated by electrode currents and molten currents in the furnace is reflected outside of the furnace. The experimental results show that the key parameters of smelting including the position of electrode tip, the length of electric arc, and the liquid level of molten bath can be achieved. The computed tomography for SAF can be realized by the detection for smelting.
\end{abstract}

Keywords : submerged arc furnace, key parameter of smelting, magnetic field detection

\section{Introduction}

Submerged arc furnace (SAF) is an industrial electric furnace which can smelt ores and extract useful metals by electric arc discharge. The electric arc occurs between furnace burden and the tip of electrodes which are the critical components that inject large current into the furnace to perform arc smelting [1]. In the process of smelting, heat distribution in furnace and balance degree of threephase power in molten bath is regulated by lifting and lowering electrodes $[2,3]$.

Three smelting parameters, including the position of electrode tip, the length of electric arc and the liquid level of molten bath, are very important for the smelting process of SAF. In most situations, the smelting parameters are achieved by simple instruments and personal experiences. However, this method is difficult to get the optimal smelting parameters which have a great influence on technical and economic parameters such as energy consumption and ore consuming. To overcome the drawback, lots of methods have been proposed on the measurement of these key parameters. Some of these methods get the parameter values through indirect calculation and specu-

(C)The Korean Magnetics Society. All rights reserved.

*Corresponding author: Tel: 132-3369-7523

Fax: 0351-6014033, e-mail: 644855751@qq.com lation [4-7]. Other methods get the position of electrode tip roughly $[8,9]$.

In order to solve the problems of low accuracy and the difficult detection for key smelting parameters in SAF, a non-contact measuring method using magnetic field analysis is put forward based on electromagnetic radiation principle in this paper. The theoretical analysis and preliminary experiments are carried out and the measuring approach can achieve the key parameters of smelting. A solution to the key parameter detection of smelting is suggested and a CT (Computed Tomography) is tailored for SAF from a new perspective.

The rest of paper is organized as follows. Section 2 introduces the theory background of the measurement. In Section 3, the proposed method is analyzed. Experimental results are presented in Section 4, and the conclusions are given in Section 5.

\section{Theory Background}

The hearth structure [10-12] of SAF is shown in Fig. 1. The electric arc is generated between electrode tip and molten bath from Fig. 1. The molten alloys gather in molten bath and the top of molten bath surface, namely liquid level.

Based on the Biot-Savart law [15], in any circuit loop at any point, the current element $I \mathrm{~d} \hat{l}^{\prime}$ is termed as source 


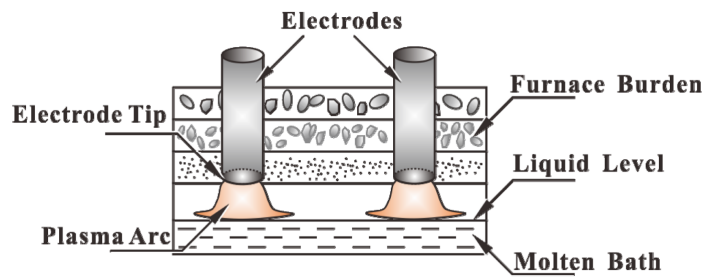

Fig. 1. (Color online) The hearth structure of SAF.

point. Those points which are needed to compute magnetic induction intensity $\vec{B}$ are called as field points. The magnetic induction intensity which is generated by current element $I \mathrm{~d} \vec{l}$ ' can be described as Eq. (1).

$$
\mathrm{d} \vec{B}(\vec{r})=\frac{\mu_{0}}{4 \pi} \frac{I \mathrm{~d} \vec{l}^{\prime} \times\left(\vec{r}-\vec{r}^{\prime}\right)}{\left|\vec{r}-\vec{r}^{\prime}\right|^{3}}
$$

where $\mu_{0}$ is the permeability of vacuum; $\vec{r}^{\prime}$ is the position vector of the source point; $\vec{r}$ is the position vector of the field point.

From Eq. (1), the magnetic induction intension generated by current-carrying straight wire with current $I$ can be expressed by the following equation:

$$
B=\frac{\mu_{0} I}{4 \pi r}\left(\cos \theta_{1}-\cos \theta_{2}\right)
$$

in which $\vec{r}_{1}$ is the distance vector from the starting point of the straight wire carrying current $I$ to the field point; $\vec{r}_{2}$ is the distance vector from the end point of the straight wire carrying current $I$ to the field point; $\theta_{1}$ is the angle between the distance vector $\vec{r}_{1}$ and the current element located at the starting point of the straight wire; $\theta_{2}$ is the angle between the distance vector $\vec{r}_{2}$ and the current element located at the end point of the straight wire.

According to the theory mentioned above, when the current value through electrodes reaches as high as 100,000 A, there inevitably exists magnetic field information reflecting the current value and direction inside the furnace. Therefore, through the available information of magnetic field distribution, the three key parameters can be obtained, including the position of electrode tip, the length of electric arc and the liquid level.

Through the electrodes, large currents flow into the furnace, in which the circuit can be regarded as two types of loop, namely star circuit loop and triangle circuit loop $[13,14]$, and the two types of loop can be equivalently transformed. In this paper we use the triangle loop as an example to analyze the circuit model. Based on the electromagnetism, magnetic field radiation model of SAF is established, as shown in Fig. 2.

In Fig. $2, I_{A}, I_{B}$, and $I_{C}$ are the currents of electrode A,

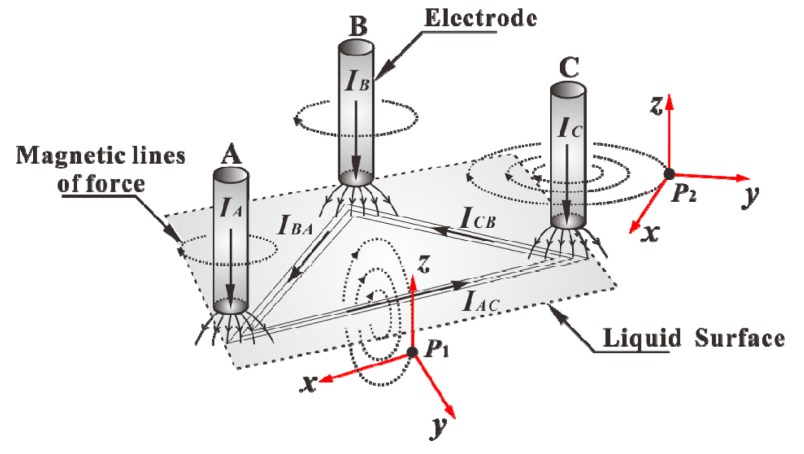

Fig. 2. (Color online) The magnetic field radiation model of SAF.

$\mathrm{B}$, and $\mathrm{C}$, respectively, and $I_{A C}, I_{C B}$, and $I_{B A}$ are the currents in the molten bath. Currents flow into the furnace along each electrode and form a triangle circuit loop near the liquid surface. The two points $P_{1}$ and $P_{2}$ are the measured origin; $I_{A C}$ is the molten bath current parallel to the $x$-axis at point $P_{1} ; I_{C}$ is the electrode current parallel to the $z$-axis at point $P_{2}$. In order to compute conveniently, the concepts of electrode line and perpendicular bisector are defined as shown in Fig. 3 .

In Fig. 3 the plan view of SAF is given. On the plane parallel to the molten bath surface, a regular triangle is generated through connecting three vertex electrodes, and the core of SAF is the center of the triangle. The line connecting the electrode and the furnace core is called electrode line. The vertical of each edge of the regular triangle is termed as perpendicular bisector. It can be seen that the perpendicular bisector is parallel to $y$-axis at point $P_{1}$, and electrode line is parallel to $y$-axis at point $P_{2}$.

The detected point $P_{1}$ in Fig. 2 is assumed to be the origin $o$, and several detection points $\left(S_{1}, S_{2}, \cdots, S_{\mathrm{m}}, \cdots\right.$, $S_{\mathrm{i}}$ ) are chosen from the $z$-axis. In these detection points, the point $S_{\mathrm{m}}$, which overlaps with the point $P_{1}$, locates on the geometric plane generated by current $I_{A C}, I_{C B}$, and $I_{B A}$. The magnetic induction intensity distribution at point $P_{1}$ in perpendicular bisector is shown in Fig. 4 under the influence of the magnetic field produced by current $I_{A C}$.

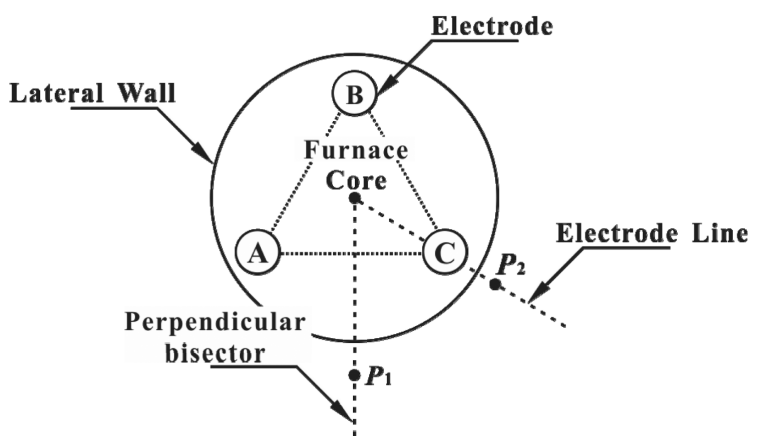

Fig. 3. The plan view of SAF. 


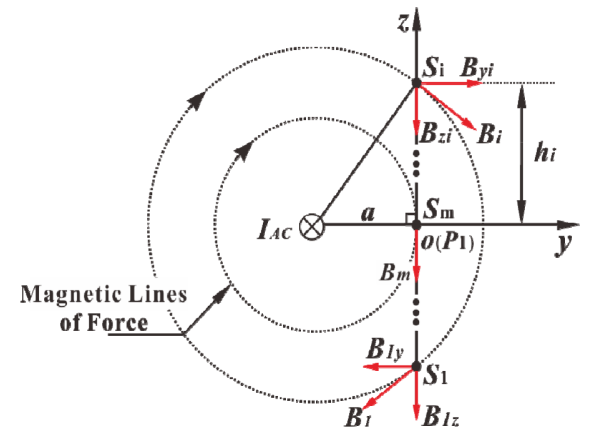

Fig. 4. (Color online) The diagram of magnetic induction intensity distribution at detected point $P_{1}$.

From Fig. 4 and Eq. (2), it can be seen that the magnetic flux density components of the detection point $S_{\mathrm{i}}$ along the $x, y$ and $z$-axis are $B_{x j}, B_{y i}$, and $B_{z i}$ which are given as Eq. (3).

$$
\begin{aligned}
B_{y i} & =\frac{\mu_{0} I_{A C} L}{2 \pi} \frac{h_{i}}{\left(a^{2}+h_{i}^{2}\right) \sqrt{L^{2}+4\left(a^{2}+h_{i}^{2}\right)}} \\
B_{x i} & =0 \\
B_{z i} & =\frac{\mu_{0} I_{A C} L}{2 \pi} \frac{a}{\left(a^{2}+h_{i}^{2}\right) \sqrt{L^{2}+4\left(a^{2}+h_{i}^{2}\right)}}
\end{aligned}
$$

where $h_{i}$ is the height of detection point $S_{\mathrm{i}} ; a$ is the distance from the origin $o$ to the pathway of current $I_{A C}$; $L$ is the length of current $I_{A C}$ in the molten bath.

Because the detection point is outside of the furnace, $\mu_{0}$ is equal to $4 \pi \times 10^{-7} \mathrm{H} / \mathrm{m}$, and $I_{A C}$ is approximately equal to $40 \mathrm{kA}[16,17]$. The bottom of the furnace is $1.3 \mathrm{~m}$ away from the ground, the height of the furnace body is around $3.7 \mathrm{~m}$, and the range of the parameter $h_{i}$ is $[-8 \mathrm{~m}$, $8 \mathrm{~m}]$. According to the environmental observation in real scene, the values of the parameter $a$ and $L$ are set to $a \approx$ $8.5 \mathrm{~m}$ and $L \approx 1.6 \mathrm{~m}$, respectively. We use the parameters mentioned above and Maple software to carry out simulation. The result is shown in Fig. 5.

From Fig. $5 B_{y i}$ is an odd function and $B_{z i}$ is an even function in the range of $[-8 \mathrm{~m}, 8 \mathrm{~m}]$. The zero point of $B_{y i}$ corresponds to the peak point of $B_{z i}$. The region which locates near the peak point of $B_{z i}$ corresponds to the current pathway of the molten bath region. We found that $B_{y i}$ and $B_{z i}$ contain the information of the liquid level in molten pool through observing the points in perpendicular bisector.

The detected point $P_{2}$ in Fig. 2 is assumed to be the origin $o^{\prime}$, and several detection points $\left(S_{1}, \cdots, S_{\mathrm{n}}, \cdots, S_{\mathrm{j}} \cdots\right)$ are chosen on the $z$-axis. $h_{j}$ is the height of the point $S_{\mathrm{j}}$, and $h_{n}$ is the height of the liquid level in molten pool. The magnetic induction intensity distribution at point $P_{2}$ on

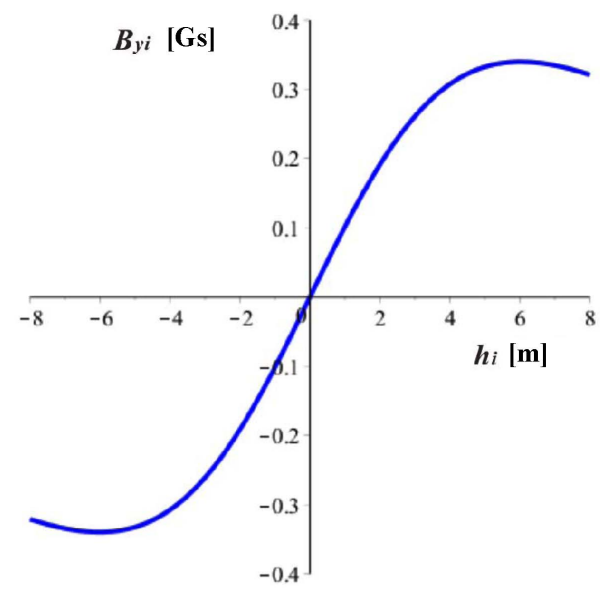

(a) $h_{i}-B_{y i}$ curve

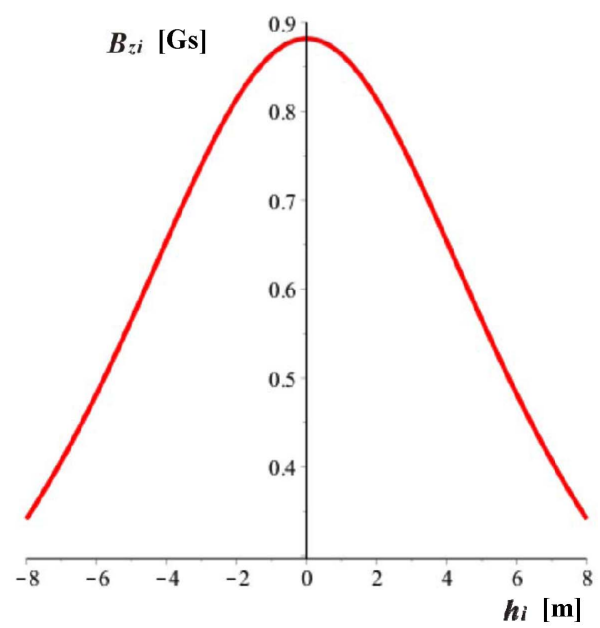

(b) $h_{i}-B_{z i}$ curve

Fig. 5. (Color online) The simulation results of magnetic flux intensity distribution at detected point $P_{1}$.

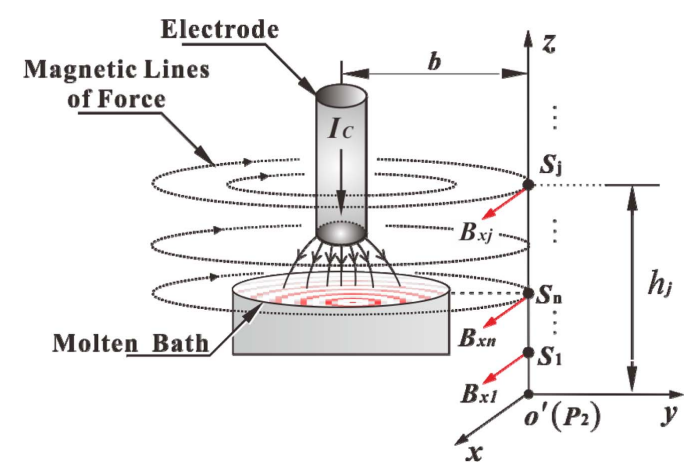

Fig. 6. (Color online) The diagram of magnetic induction intensity distribution at detected point $P_{2}$.

electrode line is shown in Fig. 6 under the influence of the magnetic field produced by the current $I_{C}$.

From Fig. 6 and Eq. (2), the magnetic flux density 
components of the detection point $S_{\mathrm{j}}$ along the $x, y$ and $z$ axis are $B_{x j}, B_{y j}$, and $B_{z j}$ which are presented as Eq. (4):

$$
\left\{\begin{array}{l}
B_{x j}=0 \quad(1 \leq j<n) \\
B_{x j}=\frac{\mu_{0}}{4 \pi} \int_{(L)} \frac{I_{C} \mathrm{~d} \vec{l} \times \vec{r}}{b^{2}} \quad(j \geq n) \\
B_{y j}=B_{z j}=0
\end{array}\right.
$$

in which $b$ is the distance from the origin $o^{\prime}$ to the pathway of current $I_{C}$. $L$ is the length of the currentcarrying straight wire with current $I_{C}$.

The currents turn to transverse direction below the liquid surface [16], so $B_{x j}=0(1 \leq j<n)$, and over the liquid surface $B_{x j} \neq 0(j \geq n)$. In this case both $B_{y j}$ and $B_{z j}$ are the constant 0 . Thus, the key parameters, including liquid level and electrode tip, can also be obtained at the point $P_{2}$.

\section{Experimental Method}

The detecting system of 3D magnetic field is composed of three identical subsystems, and the outputs of three dimensions along the $x, y$ and $z$-axis are $V_{x}, V_{y}, V_{z}$, respectively. We take the $x$-dimension as an example to explain the system in which the circuit is composed of probe, operational amplifier (OPA), low-pass filter (LPF), and RMS conversion circuit, and it is shown in Fig. 7.

In Fig. 7, the AC signal from the probe that detects magnetic field information is translated into the effective value signal as the output signal. Fig. 8 shows the scene of basic experiments.

In Fig. 8 the current-carrying coil generates magnetic

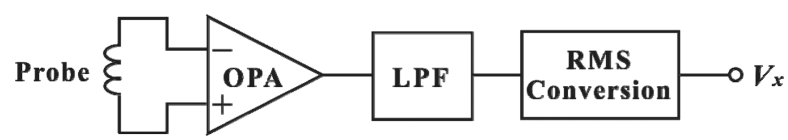

Fig. 7. Block diagram of magnetic field sensing system of $x$ axis.

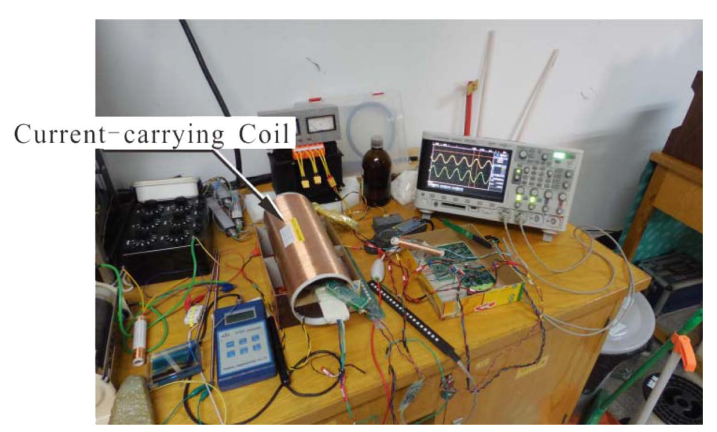

Fig. 8. (Color online) The scene of basic experiments.

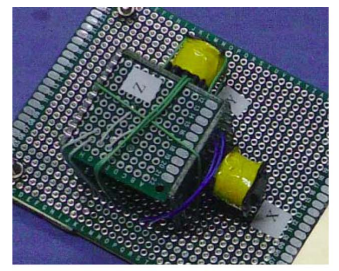

(a) Top view

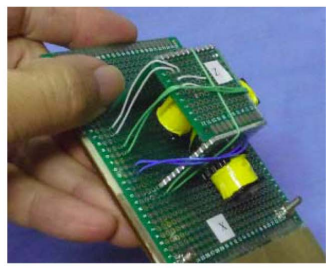

(b) Side view
Fig. 9. (Color online) The physical map of 3D probe.

field to simulate the environment of SAF in real scene. The detecting system of 3D magnetic field receives magnetic field data using 3D probe. The physical map of 3D probe is shown in Fig. 9.

We perform real measurements on the SAF of ferrosilicon manganese alloy at a metallurgical company in Yinchuan city in China. According to the theoretical analysis, the distribution of $B_{y i}$ and $B_{z i}$ in perpendicular bisector contains the information of liquid level, and the distribution of $B_{x j}$ on electrode line also contains the key information of liquid level.

After multiple experiments, we choose the test site between perpendicular bisector and electrode line, and the angle between the electrode line and the line connecting the test site and the furnace core is set to about $20^{\circ}$. We set the origin $o$ beneath the bottom of furnace which is about $6 \mathrm{~m}$ away from the lateral wall. The measuring range covers the electrode tip, which is shown in Fig. 10.

In Fig. 10, we sample 15 points along the $z$-axis for every $10 \mathrm{~cm}$ starting from the origin $o$. The measurement is repeated six times at each point, and the mean of the measurement results is taken. The experimental scene in industrial field is shown in Fig. 11.

It should be noted that the magnetic flux intensity has not been calibrated due to the limit of testing equipment. So we only present the output voltage of the system.

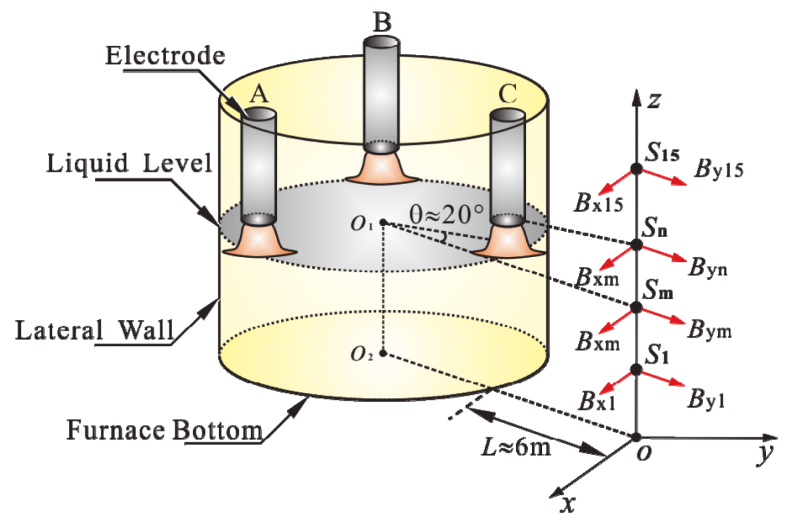

Fig. 10. (Color online) The sketch map of measuring points in real industrial field. 


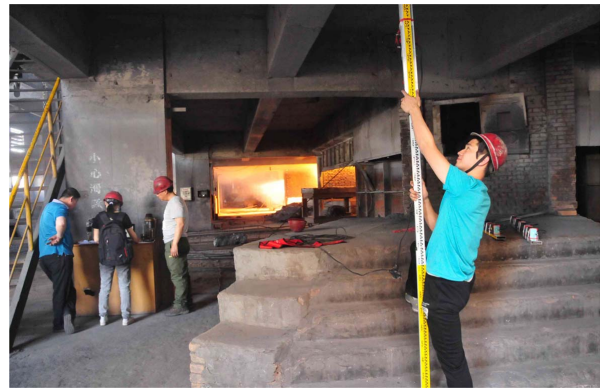

Fig. 11. (Color online) The experimental scene in industrial field.

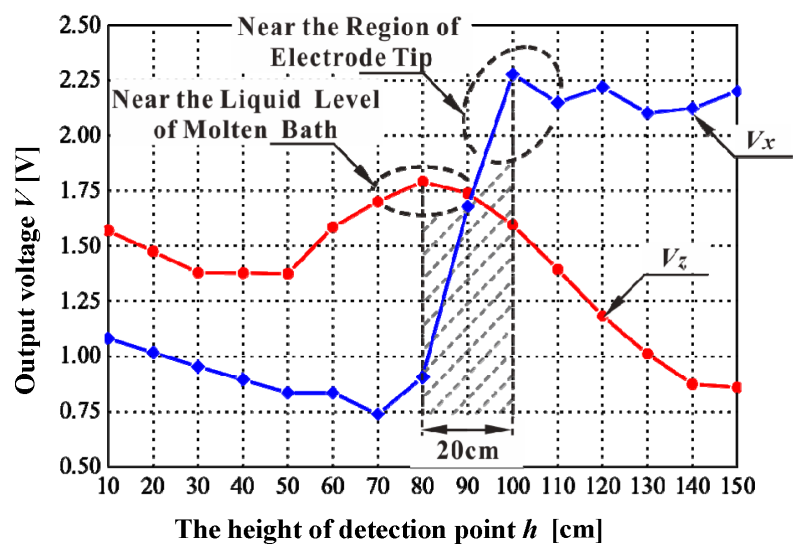

Fig. 12. (Color online) The analysis on testing results.

\section{Results and Discussion}

Due to the reason of detection point position, it is hard to get the obvious information of magnetic flux density component $B_{y}$, which makes the characteristic variation of $h \sim V_{y}$ curve less evident. Therefore, in this paper we only analyze $h \sim V_{x}$ curve and $h \sim V_{z}$ curve, as shown in Fig. 12.

From Fig. 12 it can be found that:

(1) When $h<80 \mathrm{~cm}$, the output voltage $V_{x}$ is low which indicates this section lies beneath the electrode tip; when $h>100 \mathrm{~cm}$, the output voltage $V_{x}$ is high which indicates this section lies above the electrode tip. From above, we can conclude that $h \sim V_{x}$ curve has close correlation with Eq. (4).

(2) The $h \sim V_{z}$ curve appears as a shape of bell, whose peak position shows that it lies on the same horizontal position with the transverse current $I_{A C}$. The curve of $h \sim V_{z}$ has close relation to $h_{i} \sim B_{z i}$ curve in Fig. 5 (b).

(3) It can be inferred from the two testing curves above that the electric arc beneath the electrode tip may lie in one of segments in the range of $20 \mathrm{~cm}$ shown in Fig. 12.

\section{Conclusions}

Based on electromagnetic principle, a magnetic field detection method for key smelting parameters of SAF is proposed. We prove that AC magnetic field generated by electrode currents and molten currents in the furnace is reflected outside of the furnace. And the key parameter information, such as the position of electrode tip, the length of electric arc, and the liquid level of molten bath, can be achieved by the magnetic field distribution outside of the furmace. Moreover, experimental results are consistent with the theoretical derivation on the magnetic field distribution, which illustrates the effectiveness of the proposed method.

\section{Acknowledgements}

This research has been supported by the Natural Science Foundation of Shanxi Province of China (2014011021-1).

\section{References}

[1] M. Moghadasian and E. Alenasser, J. Electromagn. Anal. Appl. 3, 47 (2011).

[2] A. C. Mulholland, P. J. Breretonstiles, and C. J. Hockaday, J. S. Afr. I. Min. Metal. 109, 601 (2009).

[3] N. N. Zhang, Z. J. Wang, and D. J. Zhang, IEEE Comput. Mechatr. Contr. Electr. Eng. 3, 108 (2010).

[4] J. Zhang, S. J. Chu, and Z. S. Li, Ferro-Alloys 3, 5 (2014).

[5] S. M. Kang, Ferro-Alloys 2, 30 (2012).

[6] J. M. Wu, Q. X. Wang, Z. Q. Xu, and Q. H. Zhu, Contr. Instru. Chem. Indus. 41, 181 (2013).

[7] Y. Wang, Z. Z. Mao, H. X. Tian, Y. Li, and P. Yuan, J. Cent. South. Univ. T. 17, 560 (2010).

[8] Y. Bai, Q. Wang, F. R. Meng, and H. Y. Wang, J. ChangChun. University T 33, 383 (2012).

[9] Z. H. An, Ms. D. Thesis, Changchun University of Technology, China (2010).

[10] S. J. Chu, S. L. Zeng, and Z. C. Huang, Ferro-Alloys 2, 13 (2009).

[11] S. J. Chu, X. E. Bao, and Z. S. Li, Ferro-Alloys 3, 22 (2013).

[12] A. S. Hauksdottir, T. Soderstrom, Y. P. Thorfinnsson, and A. Gestsson, IEEE T. Contr. Syst. T 3, 377 (1996).

[13] Q. H. Xiao, Ferro-Alloys 1, 11 (1982).

[14] N. S. Zhang, Ferro-Alloys 6, 1 (1986).

[15] Q. G. Reynolds and R. T. Jones, Miner. Eng. 19, 325 (2006).

[16] M. Ramirez, J. Alexis, G. Trapaga, P. Jonsson, and J. Mckelliget, Trans. ISIJ 19, 325 (2006).

[17] F. Martell, M. Ramirez, A. Llamas, and O. Micheloud, ISIJ International 53, 743 (2013). 